Journal of Engineering and Applied Sciences 14 (Special Issue 3): 6181-6184, 2019

ISSN: 1816-949X

(C) Medwell Journals, 2019

\title{
Antenna Design for Shallow Freshwater Applications
}

\author{
${ }^{1}$ Dzufi Iszura Ispawi, ${ }^{1,2}$ Hadi Jumaat, ${ }^{1}$ Mazlina Mansor Hassan, ${ }^{2}$ Mohd Tarmizi Ali, \\ ${ }^{3}$ Nurulazlina Ramli and ${ }^{1}$ Bibi Sarpinah Bt. Sheikh Naimullah \\ ${ }^{1}$ Faculty of Electrical Engineering, Universiti Teknologi MARA, \\ 94300 Kota Samarahan, Sarawak, Malaysia \\ ${ }^{2}$ Faculty of Electrical Engineering, Universiti Teknologi MARA, \\ 40450 Shah Alam, Selangor, Malaysia \\ ${ }^{3}$ Centre of Advanced Electrical and Electronic Systems, \\ Faculty of Engineering and the Built Environment, \\ SEGi University, 47810 Kota Damansara, Selangor, Malaysia
}

\begin{abstract}
Underwater communication technology is not as developed as free-space (terrestrial) communication. Some research are being done but most of the study's concentrating on antenna used in seawater application. An array antenna to be used in shallow water is designed. The frequency used is $405 \mathrm{MHz}$ that allowed those commercial devices with such frequency to be used in shallow freshwater in terms of communicating purpose. This research study will involve several steps which are: antenna design, simulation, producing prototype, testing prototype and result analysis. The software used for designing and simulating the antenna is CST Studio Suite. In terms of hardware, the design is by using microstrip array antenna. The advantages of this design is ease of construction, lightweight, low cost and extremely thin protrusion from surface. The key features make microstrip antenna popular in free-space applications. The result of the prototype testing will be discussed.
\end{abstract}

Key words: Underwater antenna, underwater communication, microstrip antenna, antenna array, CST Studio Suite, prototype

\section{INTRODUCTION}

It has been studied that most of the systems in communication engineering are developed to be used in free-space instead of underwater. However, in these recent years, more and more research are being done to develop a good underwater communication system (Sporer et al., 2015). Some of the applications for underwater communications are tactical network between naval platform, diver communication, autonomous underwater vehicle, ocean observatory and many more. For example, an international project called ESONET, a proposed subsea component of the European GMES (Global Monitoring for Environment and Security) to provide strategic long-term monitoring capability in Geophysics, Chemistry, Biochemistry, Oceanography, Biology and Fisheries (Mendez et al., 2011). Other examples are North America's NEPTUNE (Northeast Pacific Time-Series Undersea Networked Experiments), Japan's ARENA and Sweden's FOI (Mendez et al., 2011).
However, most of the designs are for saltwater applications. As the characteristics of saltwater is different than freshwater, different technologies are used for those applications. In saltwater, acoustic waves are used to send signal to overcome the attenuation problem (Hao et al., 2011). In shallow water, instead of acoustic wave, Electro Magnetic (EM) is used. They are unaffected by turbidity and pressure gradients and it is immune to acoustic noise (Che et al., 2010). Furthermore, the traditional underwater acoustic communication systems have been based on non-coherent detection techniques such as FSK which have high reliability rather than bandwidth efficiency (Chavhan and Sarate, 2013).

Previously, EM never been chosen for underwater communication solution (Sendra et al., 2013). This is because the high signal attenuation due to the conductivity of the water. However, the main advantages of EM waves compare to acoustic waves is it do not need the movements of particles of medium to be propagated

Corresponding Author: Dzufi Iszura Ispawi, Faculty of Electrical Engineering, Universiti Teknologi MARA, 
(Hao et al., 2011; Sendra et al., 2013). Underwater communications based on EM waves are faster and can be used in higher working frequencies which results in a higher bandwidth.

For the research, it is proved that EM is suitable for higher frequencies as the proposed design is to be work at $410 \mathrm{MHz}$. Previous research shown that there are three unlicensed frequencies work best for underwater communication system which are: $6.7 \mathrm{MHz}, 433 \mathrm{MHz}$ and $2.4 \mathrm{GHz}$ (Abdou et al., 2011). The $2.4 \mathrm{GHz}$ are chosen because the frequency is widely used for $\mathrm{WiFi}$, internet and Bluetooth connection.

Different types of antenna are already being tested for freshwater applications in previous research. Curved spiral antenna is mounted on pocketbook mussel (Lampsilis cardium) in river water (Llamas et al., 2015). For a spiral antenna, it is the combination of its plane geometry and location of differential radiating elements in that plane that leads to its broadband characteristics. Thus, if one disturbs the location of these radiating elements, these properties are compromised (Llamas et al., 2015). A vibrator antenna and a loop antenna are also being tested. Although, a vibrator antenna may have a reduced signal to noise ratio, it can be employed over greater distances (Hao et al., 2011).

A microstrip array design is chosen because of its advantage. Microstrip antennas attractive features such as low profile, light weight, small volume and low production cost (Sabban, 2010). In addition, integrating the microstrip feed structure with the radiating elements on the same substrate attains the benefit of a compact low cost feed network. However, losses in the microstrip feed network form a significant limit on the possible applications of microstrip antenna arrays in $\mathrm{mm}$ wave frequency range (Milkov, 2000).

\section{MATERIALS AND METHODS}

Antenna design: Microstrip is used for the proposed antenna (Fig. 1). The advantages of this design is ease of construction, lightweight, low cost and extremely thin protrusion from surface. The key features make microstrip antenna popular in free-space applications. The material was tested to be able to function in freshwater with the used of buffer. Buffer for the design of proposed antenna is liquid with dielectric constant $\left(\varepsilon_{\mathrm{r}}\right)$ in range of 7-8. Aniline $\left(\mathrm{C}_{6} \mathrm{H}_{7} \mathrm{~N}\right)$ with dielectric constant value 7.8 is used for the prototype. The detailed geometry of proposed antenna is shown in (Table 1).
Table 1: Geometry of proposed antenna

\begin{tabular}{lc} 
Geometry & Centimeter $(\mathrm{cm})$ \\
\hline Length (antenna) & 16 \\
Width (antenna) & 17 \\
Length (feeder) & 12 \\
Width (feeder) & 0.2 \\
\hline
\end{tabular}

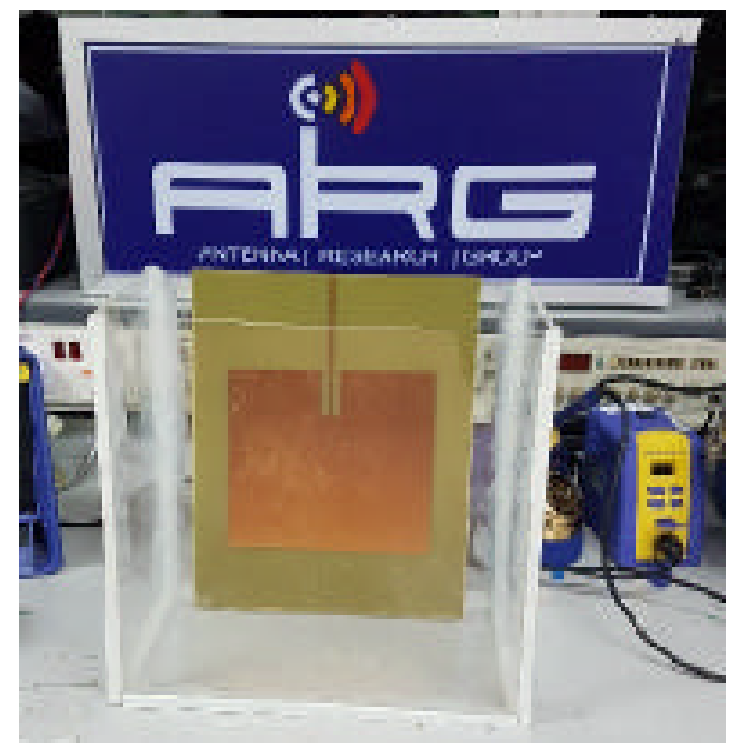

Fig. 1: Prototype of the antenna design

\section{RESULTS AND DISCUSSION}

The prototype was tested in three environments; free space (air) in water without buffer and in water with buffer. The simulation result of the antenna was carried out via. CST microwave studio which is a full wavelength numeric electromagnetic simulation tool. Figure $2 \mathrm{a}, \mathrm{b}$ show the testing done in water environment. The S11 parameters for the antenna for each environments were tested using FieldFox microwave analyser.

For free space (air) environment, the test showed that the antenna was able to function but not in wanted frequency. Both measured and simulated frequencies are shifted to higher frequency. The measured result was $490 \mathrm{MHz}$ meanwhile the simulated frequency was $460 \mathrm{MHz}$. Figure 3a shows the S11 parameters for the prototype in free air environment.

No attainable result produced when the prototype tested directly inside water without buffer as in Fig. 3b. It shows that the prototype was not complete and could not function without buffer inside water. When aniline is used as buffer for the prototype, the result obtained as in Fig. 4. The measured result for the prototype was $405 \mathrm{MHz}$. 
(a)

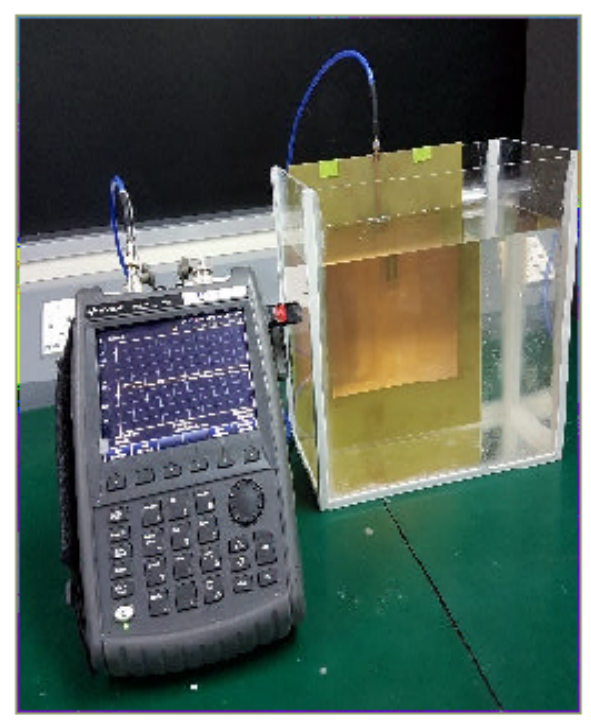

(b)

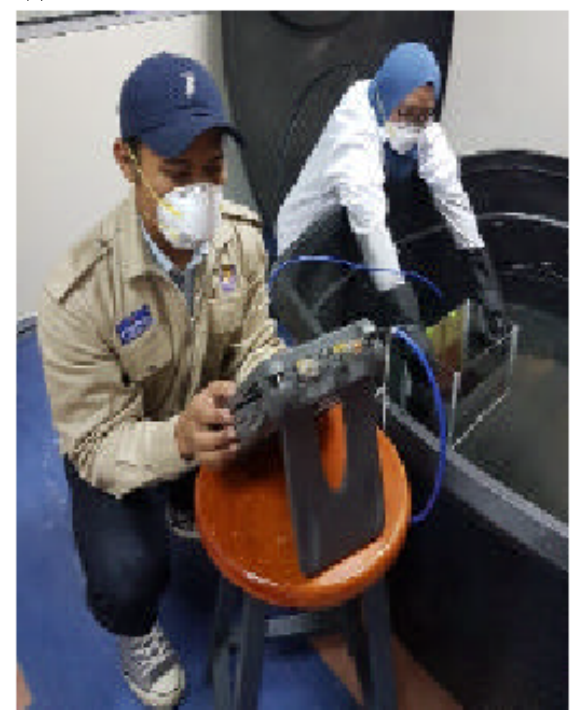

Fig. 2: a) Testing prototype without buffer in water and b) Testing prototype with buffer
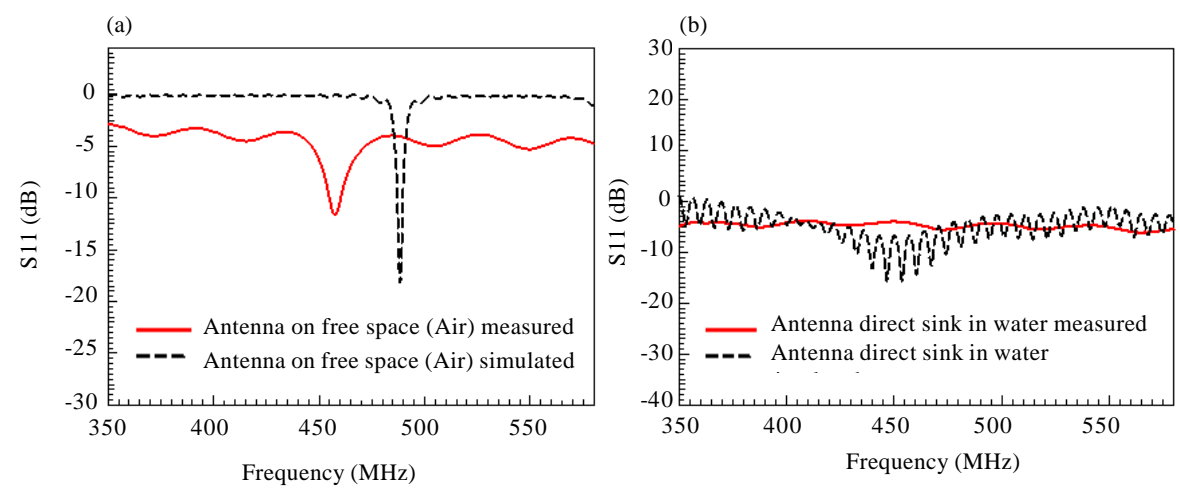

Fig. 3: a) S-parameter antenna on free space (air) and b) S-parameter antenna inside water without buffer

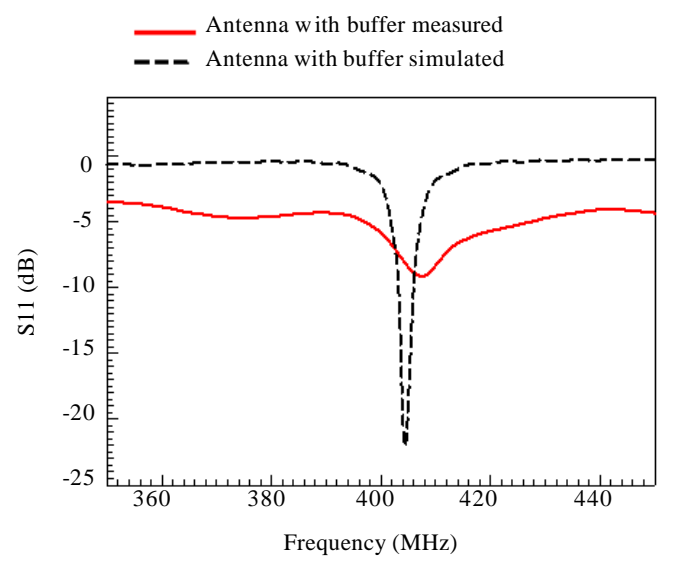

Fig. 4: S-parameter antenna with buffer inside water

\section{CONCLUSION}

The proposed design for the antenna is able to function in shallow freshwater in $405 \mathrm{MHz}$ frequency. The choice of buffer used play significant role in order for the prototype to function. Further testing is needed to obtain the radiation pattern to conclude the effectiveness of the proposed antenna design.

\section{ACKNOWLEDGEMENT}

This research was supported by the Faculty of Electrical Engineering (Antenna Research Group) and UiTM. The researchers also wish to thank UiTM Sarawak (Grant Dana Kecemerlangan) for the financial aid for this project. 


\section{REFERENCES}

Abdou, A.A., A. Shaw, A. Mason, A. Al-Shamma'a and J. Cullen et al., 2011. Electromagnetic (EM) wave propagation for the development of an underwater Wireless Sensor Network (WSN). Proceedings of the 2011 IEEE International Conference on SENSORS, October 28-31, 2011, EEE, Limerick, Ireland, ISBN: 978-1-4244-9290-9, pp: 1571-1574.

Chavhan, J.W. and G.G. Sarate, 2013. Smart Antenna approach in underwater Acoustic Sensor Network using OFDM: A review. Proceedings of the 2013 International Conference on Green Computing, Communication and Conservation of Energy (ICGCE), December 12-14, 2013, IEEE, Chennai, India, ISBN: 978-1-4673-6126-2, pp: 155-158.

Che, X., I. Wells, G. Dickers and P. Kear, 2010. Re-evaluation of RF electromagnetic communication in underwater sensor networks. IEEE. Commun. Mag., 48: 143-151.

Hao, Z., G. Dawei, Z. Guoping and T.A. Gulliver, 2011. The impact of antenna design and frequency on underwater wireless communications. Proceedings of 2011 IEEE International Pacific Rim Conference on Communications, Computers and Signal Processing, August 23-26, 2011, IEEE, Victoria, Canada, ISBN: 978-1-4577-0252-5, pp: 868-872.
Llamas, R.A., J.J. Niemeier and A. Kruger, 2015. Curved spiral antennas for freshwater applications. Proceedings of the 2015 IEEE International Symposium on Radio and Wireless (RWS), January 25-28, 2015, IEEE, SanDiego, California, USA., ISBN: 978-1-4799-5507-7, pp: 53-55.

Mendez, H.F.G., C. Gac, F. Le Pennec and C. Person, 2011. High performance underwater UHF radio antenna development. Proceedings of the IEEE Spain Conference on OCEANS 2011, June 6-9, 2011, EEE, Santander, Spain, ISBN: 978-1-4577-0086-6, pp: 1-5.

Milkov, M.M., 2000. Millimeter-wave imaging system based on antenna-coupled bolometers. MSc Thesis, University of California, Los Angeles, Los Angeles, California

Sabban, A., 2007. Applications of MM wave microstrip antenna arrays. Proceedings 2007 International Symposium on Signals, Systems and Electronics, July 30-August 2, 2007, IEEE, Montreal, Canada, ISBN:1-4244-1 448-2, pp: 119-122.

Sendra, S., J. Lloret, J.J.P.C. Rodrigues and J.M. Aguiar, 2013. Underwater wireless communications in freshwater at $2.4 \mathrm{GHz}$. IEEE. Commun. Lett., 17: 1794-1797.

Sporer, M., F. Lurz, E. Schluecker, R. Weigel and A. Koelpin, 2015. Underwater interferometric radar sensor for distance and vibration measurement. Proceedings of the 2015 IEEE International Topical Conference on Wireless Sensors and Sensor Networks (WiSNet), January 25-28, 2015, IEEE, San Diego, California, USA., ISBN:978-1-4799-5519-0, pp: 72-74. 